\title{
Low-Complexity Iterative Quantum Multi-User Detection in SDMA systems
}

\author{
Panagiotis Botsinis, Soon Xin Ng, and Lajos Hanzo \\ Electronics and Computer Science, University of Southampton, SO17 1BJ, United Kingdom. \\ Email: $\{$ pb8g10, sxn, lh\}@ecs.soton.ac.uk, www-mobile.ecs.soton.ac.uk
}

\begin{abstract}
The potentially excessive complexity of the Maximum Likelihood Multi-User Detector (ML MUD) in largescale Spatial Division Multiple Access (SDMA) systems dictates the employment of low-complexity sub-optimal MUDs in the context of conventional systems. However, this limitation was circumvented by the recently proposed Dürr-Høyer Algorithm (DHA)-aided Quantum Weighted Sum Algorithm (QWSA)-based Quantum Multi-User Detector (QMUD) employed for performing optimal ML iterative detection in SDMA systems. Focusing our attention on the QWSA, we analyse the QMUD and the evolution of the quantum system with the aid of a simple SDMA uplink scenario. We characterize the performance of the DHA-QWSA QMUD advocated, which is capable of matching the performance of the ML MUD both in terms of its EXIT charts and BER curves.
\end{abstract}

Index Terms-Computational Complexity, Quantum Computing, Quantum Search Algorithms, Spatial Division Multiple Access.

\section{INTRODUCTION}

The complexity of the optimal Maximum Likelihood MultiUser Detector (ML MUD) conceived for Spatial Division Multiple Access (SDMA) systems increases exponentially both with the number of users $K$, with the number of transmit antennas $L$ per user, as well as with the number of bits $m$ per modulated symbol [1]. By exploiting the parallel nature of quantum computing [2]-[4], Imre and Balázs proposed a hardinput hard-output QMUD for CDMA systems [3], [5], whilst achieving a substantial computational complexity reduction compared to the classic ML MUD.

For performing optimal soft-input soft-output lowcomplexity iterative detection in SDMA systems we may use the Quantum Weighted Sum Algorithm (QWSA) QMUD of [6]. The QWSA estimates the weighted sum of a function $f:\left\{0,1, \ldots, 2^{n}-1\right\} \rightarrow[0,1]$ and it is based on the Quantum Mean Algorithm (QMA) of [7]. The Dürr-Høyer Algorithm (DHA) of [8], which is based on Grover's Quantum Search Algorithm (QSA) [9] and on the Boyer-Brassard-Høyer-Tapp (BBHT) QSA of [10], is employed here for finding the particular multi-user symbol that minimizes a specific Cost Function (CF) involved in the QWSA [6].

In this treatise our novel contributions are

1) An analysis of the QWSA based on a simple SDMA uplink scenario, offering deeper intuition on the DHA-

The financial support of the RC-UK under the India-UK Advanced Technology Centre (IU-ATC), of the EU under the Concerto project and that of the European Research Council, Advanced Fellow Grant and of the Royal Society's Wolfson Research Merit Award is gratefully acknowledged.
QWSA QMUD, while providing the formulas for the quantum system's evolution.

2) The application of the DHA-QWSA QMUD in the uplink of SDMA systems, where each user is assumed to have multiple transmit antennas (TA), while multiple receive antennas (RA) are installed at the Base Station (BS).

3) We compare the DHA-QWSA QMUD's performance to that of the ML MUD both with the aid of EXtrinsic Information Transfer (EXIT) charts $^{1}$ and BER curves.

The rest of the paper is structured as follows. Section II details the model of an SDMA communication system and presents the specific scenario considered for the analysis of the QWSA in Section III, where the theoretical background required on quantum computing is also provided. The complexity and simulation results of the DHA-QWSA QMUD in a larger SDMA system are offered in Section IV. Finally, our conclusions are given in Section $\mathrm{V}$.

\section{SySTEM OVERVIEW}

The block diagram of an SDMA system's uplink supporting $K$ users with the aid of $L$ TAs and $P$ RAs at the BS is shown in Fig. 1. The bit stream of the $k$ th user $\left\{b_{k}\right\}$ is encoded using Turbo Convolutional Codes (TCC) into the bit stream $\left\{c_{k}\right\}$, before it is passed through the bit-based interleavers resulting in $\left\{u_{k}\right\}$. The modulated symbol stream $\left\{x_{k}\right\}$ is split in $L$ parallel streams and it is transmitted over uncorrelated Rayleigh channels. The channel matrix $\mathbf{H}$ is assumed to be perfectly estimated at the BS. In our scenario we will investigate an SDMA system supporting $K=2$ users with the aid of $L=1$ TA each, employing BPSK modulation $(M=2)$ and $P=1 \mathrm{RA}$ at the BS, as seen in Table I.

Assuming a synchronous system, the $(K \cdot L)$ signals at the $p$ th RA are superimposed and the thermal noise is added at each antenna. The $P$ signals are passed to the QMUD as in

$$
\mathbf{r}=\mathbf{H} \mathbf{x}+\mathbf{n},
$$

where $\mathbf{r}=\left[r_{0}, \ldots, r_{P-1}\right]^{T}$ includes each receive antenna's output during the same time slot, $\mathbf{H} \in \mathbb{C}^{P \times K \cdot L}$ contains the channel coefficients, $\mathbf{x}=\left[x_{0}, \ldots, x_{K-1}\right]^{T}$ is the transmitted $\left(M^{K \cdot L}\right)$-ary multi-level symbol, while $\mathbf{n}=\left[n_{0}, \ldots, n_{P-1}\right]^{T}$ is the Additive White Gaussian Noise (AWGN) imposed on each RA.

\footnotetext{
${ }^{1}$ For a tutorial on EXIT charts, please refer to [11].
} 


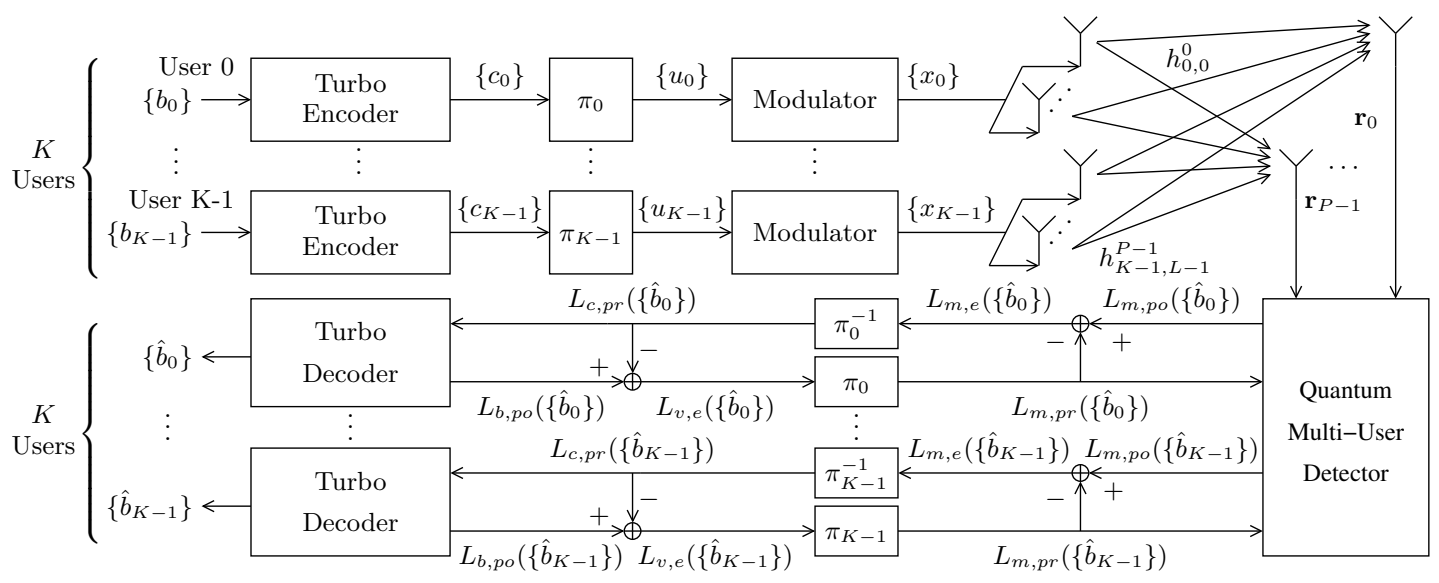

Fig. 1. SDMA uplink communication system's block diagram supporting $K$ users with $L$ transmit antennas each, employing turbo encoding using two convolutional codes as well as iterative QMUD at the BS, where $P$ receive antennas are installed.

TABLE I

PARAMETERS OF THE SDMA UPLINK SCENARIO

\begin{tabular}{ll}
\hline Number of Users & $K=2$ \\
Number of TAs per User & $L=1$ \\
Number of RAs at the BS & $P=1$ \\
Modulation & BPSK $M=2$ \\
Channel Code & TCC, $R=1 / 2,8$ trellis states \\
& $I_{\text {inner }}=4$ iterations \\
$E_{b} / N_{0}$ & $0 \mathrm{~dB}$ \\
CSI: $\mathbf{H}=\left[h_{0,0}^{0} h_{1,0}^{0}\right]$ & $h_{0,0}^{0}=0.067+j 0.62$ \\
& $h_{1,0}^{0}=0.75+j 0.32$ \\
Coded \& Interleaved Bits & $u_{0}=0, u_{1}=1$ \\
A Priori Bit Probabilities & $P\left(b_{0,0}^{(0)}=0\right)=0.5$ \\
& $P\left(b_{1,0}^{(0)}=0\right)=0.25$ \\
\hline
\end{tabular}

The QMUD initially finds the specific $\left(M^{K \cdot L}\right)$-ary symbol $\mathbf{x}_{\text {max }}$ that maximizes the $\mathrm{CF}$

$$
f(\mathbf{x})=P(\mathbf{r} \mid \mathbf{x})=\exp \left(-\|\mathbf{r}-\mathbf{H} \mathbf{x}\|^{2} / 2 \sigma^{2}\right)
$$

where $\sigma^{2}$ is the noise variance. This is achieved with an $\sim$ $100 \%$ probability by employing the DHA [8], after evaluating the CF of (2) $O\left(\sqrt{M^{K \cdot L}}\right)$ times in the Quantum Domain (QD) as detailed in [6]. The complexity of a single Cost Function Evaluation (CFE) in the QD will depend on the specific technology employed, hence for simplicity we assume that a single CFE in the QD has the same complexity as a single CFE in the Classic Domain (CD) [2], [3]. Subsequently, the QMUD computes the bit-based Log Likelihood Ratios (LLR) of the a posteriori information of the encoded, interleaved bits as encapsulated in

$$
L_{m, p o}\left(b_{k, l}^{(m)}\right)=\log \frac{\sum_{\mathbf{x} \in \chi(k, l, m, 0)} P(\mathbf{x}) f(\mathbf{x}) / f\left(\mathbf{x}_{\max }\right)}{\sum_{\mathbf{x} \in \chi(k, l, m, 1)} P(\mathbf{x}) f(\mathbf{x}) / f\left(\mathbf{x}_{\max }\right)},
$$

where the subscripts $k$ and $l$ denote the specific user $k \in$
TABLE II SDMA SCENARIO's COST FunCTION EVALUATIONS

\begin{tabular}{|c|c||c|c|}
\hline$i$ & $P(\mathbf{r} \mid x=i)=f(i)$ & $i$ & $P(\mathbf{r} \mid x=i)=f(i)$ \\
\hline 0 & 0.082 & 2 & 0.107 \\
\hline 1 & 0.811 & 3 & 0.393 \\
\hline
\end{tabular}

TABLE III

SDMA SCENARIO'S LOG LIKELIHOOD RATIOS

\begin{tabular}{|c|c|c|r|}
\hline$(k, l, m)$ & $P\left(b_{k, l}^{(m)}=0 \mid \mathbf{r}\right)$ & $P\left(b_{k, l}^{(m)}=1 \mid \mathbf{r}\right)$ & $L\left(b_{k, l}^{(m)}\right)$ \\
\hline$(0,0,0)$ & 0.629 & 0.321 & 0.673 \\
\hline$(1,0,0)$ & 0.094 & 0.602 & -1.857 \\
\hline
\end{tabular}

$\{0, \ldots, K-1\}$ and TA $l \in\{0, \ldots, L-1\}$ the bit belongs to, while the superscript $m \in\left\{0, \ldots, \log _{2}(M)-1\right\}$ is the index of that particular symbol's bit, which the LLR is computed for. Moreover, $\chi(k, l, m, \mathrm{v})$ is the set of $\left(M^{K \cdot L}\right)$-ary symbols for which the $\left(k \cdot L \log _{2}(M)+l \cdot \log _{2}(M)+m\right)$ th bit is equal to $\mathrm{v}$ and $P(\mathbf{x})$ is the a priori probability of the symbol $\mathbf{x}$. Since the bits in a symbol are assumed to be independent of each other, the $a$ priori probability of a symbol is equal to the product of the a priori probabilities of the bits it consists of.

The extrinsic LLRs at the output of the QMUD are deinterleaved and fed to the Max-Log A Posteriori Probability (APP) iterative channel decoders [11]. The extrinsic LLRs of the channel decoders are interleaved and passed back to the QMUD. After a predetermined number of QMUD-decoder iterations is performed, the information bit stream $\left\{\hat{b}_{k}\right\}$ is estimated based on the a posteriori LLRs at the output of the $k$ th decoder.

In our notation, we will replace the multi-level symbol $\mathbf{x}$ with its decimal representation $x \in\left\{0, \ldots, M^{K \cdot L}-1\right\}$. For example, since in our scenario we have $u_{0}=0, u_{1}=1$ and Gray mapping is used, then $\mathbf{x}=[+1,-1]^{T}$ demodulates into [0|1] and $x=1$. Tables II and III show the CFEs of the $M^{K \cdot L}$-ary symbols and the LLRs the two bits in our scenario, respectively, as obtained by the ML MUD. 


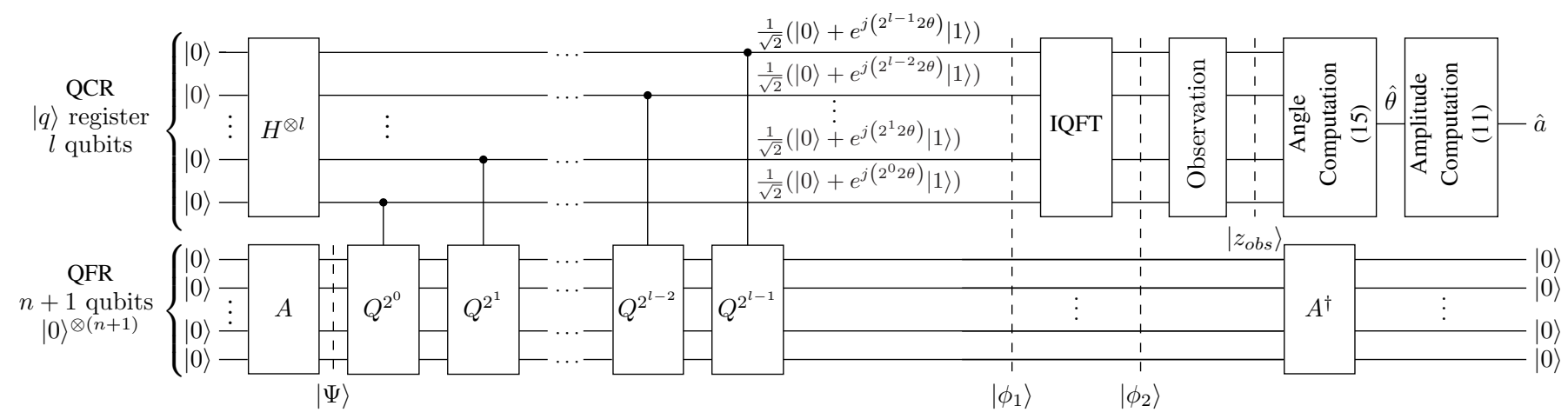

Fig. 2. Quantum circuit of the QWSA, where the quantum states $|\Psi\rangle,\left|\phi_{1}\right\rangle$ and $\left|\phi_{2}\right\rangle$ are described in (10), (12) and (13), respectively. The superscripts of the $Q$ operators signify the number of times the $Q$ operator will be consecutively applied. The number of qubits in the $\mathrm{QFR}$ is $(n+1)=\left(K \cdot L \log _{2} M-1+1\right)=$ $K \cdot L \log _{2} M$.

\section{QuANTUM ALGORITHMS}

A quantum bit or qubit $|q\rangle$ is the QD equivalent of the classic bit [2], which has the property that it may be found in any superposition of the quantum states $|0\rangle$ and $|1\rangle$ with amplitudes $a \in \mathbb{C}$ and $b \in \mathbb{C}$, respectively, as in $|q\rangle=a|0\rangle+b|1\rangle$. After an observation ${ }^{2}$ of $|q\rangle$ in the computational basis $\{|0\rangle,|1\rangle\}$ we will acquire $|q\rangle=|0\rangle$ or $|q\rangle=|1\rangle$ with probability $|a|^{2}$ and $|b|^{2}$, respectively. The quantum state $|q\rangle$ evolves with the application of unitary operators $U$, such as the Hadamard operator $H$, which maps $|0\rangle$ to $|+\rangle=\frac{1}{\sqrt{2}}(|0\rangle+|1\rangle)$ and $|1\rangle$ to $|-\rangle=\frac{1}{\sqrt{2}}(|0\rangle-|1\rangle)$.

\section{A. Quantum Search Algorithms}

Given a known value $\delta$, Grover's QSA may find the unique $S=1$ solution $x$ satisfying $f(x)=\delta$ in the set of $N$ legitimate values of $x$ with $\sim 100 \%$ success probability after $O(\sqrt{N})$ CFEs in the QD [9]. If multiple solutions exist, i.e. we have $S>1$, the BBHT QSA succeeds in finding one of them after $O(\sqrt{N / S})$ CFEs in the QD, based on Grover's QSA [10]. Finally, if the specific argument $x_{\min }$ that minimizes the CF has to be determined, but $f\left(x_{\min }\right)$ is not known a priori - as it is the case in practical applications then the DHA manages to find $x_{\min }$ after $O(\sqrt{N})$ CFEs in the QD, albeit a minimum of $4.5 \sqrt{N}$ CFEs have to be carried out [8] by repeatedly employing an appropriate version of the BBHT QSA, where the indices $x$ that satisfy $f(x)<\delta$ are marked as solutions ${ }^{3}$ [8]. Before commencing with the QWSA analysis, the DHA will be invoked for finding $x_{\max }=1$ by searching for the specific index that minimizes $-f(x)$ of (2). The solution $x_{\max }=1$ was obtained after 10 CFEs in the QD and $f\left(x_{\max }\right)=0.775$ was computed. It should be noted that the DHA is employed only once per symbol, regardless of the number $J$ of MUD-decoder iterations, since $f\left(x_{\max }\right)$ is independent of the a priori probabilities.

\section{B. Quantum Weighted Sum Algorithm}

The quantum circuit of the QWSA is presented in Fig. 2, whilst the associated procedure is summarized in Algorithm 1.

\footnotetext{
${ }^{2}$ This is reminiscent of a spinning coin in a box, which may be assumed to be both Head or Tails, until the box is opened and the coin is observed.

${ }^{3}$ For a tutorial on Grover's QSA, BBHT QSA and DHA, please refer to [6].
}

Algorithm 1: Quantum Weighted Sum Algorithm

1: Resources: For estimating the weighted sum $a$ of a function $f:\left\{0,1, \ldots, 2^{n}-1\right\} \rightarrow[0,1]$, set $l$ qubits for the QCR in the all-zero state $|q\rangle=|0\rangle^{\otimes l}$ and $n+1$ qubits for the QFR in the all-zero state $|0\rangle^{\otimes(n+1)}$.

2: Initialization: The QCR passes through a Hadamard gate $H^{\otimes l}$ as in (5), while the $A$ operator (6), (8) is applied on the QFR resulting in the state $|\Psi\rangle$ described in (10). The wanted weighted sum $a$ is the sum of the probabilities of observing half of the quantum states that $|\Psi\rangle$ is superimposed in, namely the probability of observing a state belonging to $\left|\Psi_{1}\right\rangle$.

3: Controlled $Q$ operators: The QCR controls a number of $Q$ operators with the QFR acting as the target QR. The QCR's qubits determine the specific number of $Q$ operators that are applied on the QFR each subsequent time. The resultant state of the system $\left|\phi_{1}\right\rangle$ in Figure 2 is presented in (12).

4: Inverse Quantum Fourier Transform: The IQFT is applied on the QCR, yielding the quantum state $\left|\phi_{2}\right\rangle$ described in (13).

5: Observation of the QCR: The QCR is observed and the state $\left|z_{o b s}\right\rangle \in\left\{|0\rangle,|1\rangle, \ldots,\left|2^{l}-1\right\rangle\right\}$ is obtained with the probability given in (14).

6: Estimation of $\theta$ : Based on $\left|z_{o b s}\right\rangle$ and (15), $\hat{\theta}$ is found.

7: Estimation of $a$ : Based on $\hat{\theta}$ and (11), $\hat{a}$ is computed.

In our QWSA QMUD scenario, we estimate the numerator of the first user's first bit's extrinsic LLR, which based on (3) is

$$
P\left(b_{1,0}^{0}=0\right) \cdot f^{\prime}(0)+P\left(b_{1,0}^{0}=1\right) \cdot f^{\prime}(1),
$$

where we introduced the notation $f^{\prime}(x)=f(x) / f\left(x_{\max }\right)=$ $f(x) / f(1)$.

1) Resources: The Quantum Function Register (QFR) consists of $(n+1)$ qubits, where $n$ is the number of bits in the $\left(M^{K \cdot L}\right)=2$-ary symbol minus one, since the bit we want to compute the LLR for is fixed to 0 . Hence, we need $n=K \cdot L \cdot \log _{2}(M)-1=1$ qubit to represent the $2^{n}=2$ CFEs that appear in the numerator of (3) and the QFR will have $n+1=2$ qubits in total, superimposed in the $N=2^{n+1}=4$ element all-zero state ${ }^{4}|0\rangle^{\otimes 2}$. The Quantum Control Register

\footnotetext{
${ }^{4}$ The $Z$-element tensor product is defined as: $|0\rangle^{\otimes Z}=$ $\underbrace{|0\rangle_{1} \otimes|0\rangle_{2} \otimes \cdots \otimes|0\rangle_{Z-1}}_{Z}=\underbrace{|0\rangle_{1}|0\rangle_{2} \ldots|0\rangle_{Z-1}}_{Z}=\underbrace{|00 \ldots 0\rangle}_{Z}$
} 

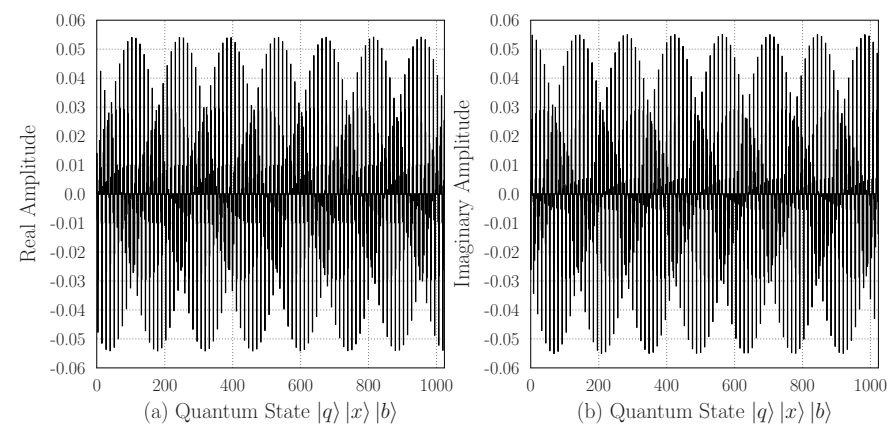

Fig. 3. The (a) real and (b) imaginary part of the amplitudes of the computational basis's quantum states $\left|\phi_{1}\right\rangle$ is superimposed in.

(QCR) consists of $l=8$ qubits, also in the all-zero state $|0\rangle^{\otimes 8}$.

2) Initialization: Initially, an $l$-qubit Hadamard gate $H^{\otimes l}$ is applied to the QCR, resulting in an equiprobable superposition of all $2^{8}$ states as in

$$
|q\rangle=H^{\otimes l}|0\rangle^{\otimes l}=\frac{1}{\sqrt{2^{l}}} \sum_{\psi=0}^{2^{l}-1}|\psi\rangle=\frac{1}{16} \sum_{\psi=0}^{255}|\psi\rangle .
$$

Each of the first $n=1$ qubits of the QFR is rotated by a qubit-specific rotation gate having an angle, which depends on the a priori probability of its corresponding bit, as in [6]

$$
R_{i}|0\rangle_{i}=\sqrt{P\left(b_{k, l}^{(m)}=0\right)}|0\rangle+\sqrt{P\left(b_{k, l}^{(m)}=1\right)}|1\rangle,
$$

where the $i=\left(k \cdot L \cdot \log _{2}(M)+l \cdot \log _{2}(M)+m\right)$ th rotation gate is described. Therefore, the initialized QFR is in the state:

$$
|\xi\rangle=\sum_{x=0}^{N-1} \sqrt{P(x)}|x\rangle|0\rangle=\sqrt{0.25}|0\rangle|0\rangle+\sqrt{0.75}|1\rangle|0\rangle .
$$

A unitary operator $A^{\prime}$ is then applied to $|\xi\rangle$ of (7), where the operation of $A^{\prime}$ may be described as [6], [7]

$$
|x\rangle|0\rangle \stackrel{A^{\prime}}{\longrightarrow}|x\rangle\left(\sqrt{1-f^{\prime}(x)}|0\rangle+\sqrt{f^{\prime}(x)}|1\rangle\right),
$$

and in our scenario the resultant state $|\Psi\rangle=A^{\prime}|\xi\rangle$ is

$$
\begin{aligned}
|\Psi\rangle & =\sqrt{0.25}|0\rangle\left(\sqrt{1-f^{\prime}(0)}|0\rangle+\sqrt{f^{\prime}(0)}|1\rangle\right) \\
& +\sqrt{0.75}|1\rangle\left(\sqrt{1-f^{\prime}(1)}|0\rangle+\sqrt{f^{\prime}(1)}|1\rangle\right) \\
& =(0.474|0\rangle+0|1\rangle)|0\rangle+(0.159|0\rangle+0.866|1\rangle)|1\rangle .
\end{aligned}
$$

We note that the probability of observing the last qubit of the QFR in the state $|1\rangle$ is equal to $a=0.159^{2}+$ $0.866^{2}=0.775$, which is equal to the desired weighted sum $P\left(b_{0,0}^{(0)}=0 \mid \mathbf{r}\right) / f\left(x_{1}\right)=0.629 / 0.811$ of Table III. In the general case, applying the operator $A=A^{\prime}\left(R_{0} \otimes \cdots \otimes R_{n-1} \otimes I\right)$ to the all-zero state $|0\rangle^{\otimes n+1}$ results in [6]

$$
|\Psi\rangle=\underbrace{\sum_{x=0}^{N-1} \sqrt{P(x)(1-f(x))}|x\rangle|0\rangle}_{\sqrt{1-a}\left|\Psi_{0}\right\rangle}+\underbrace{\sum_{x=0}^{N-1} \sqrt{P(x) f(x)}|x\rangle|1\rangle}_{\sqrt{a}\left|\Psi_{1}\right\rangle},
$$

where $a=\sum_{x=0}^{N-1} P(x) f(x)$ is the desired weighted sum, while $\left|\Psi_{1}\right\rangle$ and $\left|\Psi_{0}\right\rangle$ include the wanted and unwanted states,
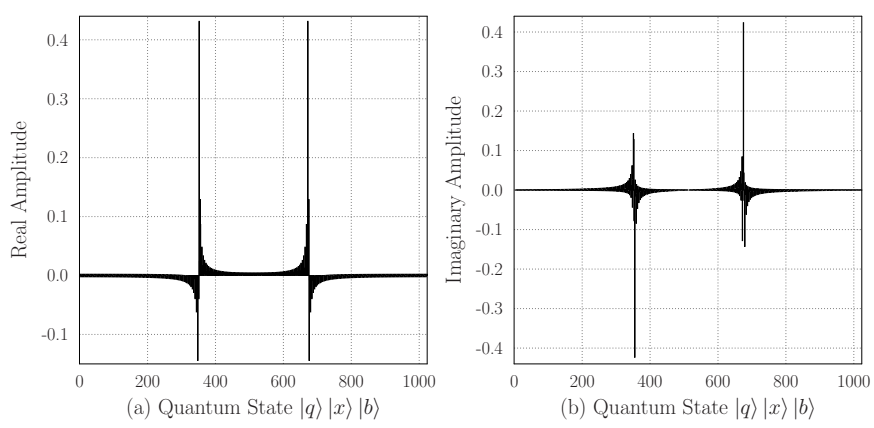

Fig. 4. The (a) real and (b) imaginary part of the amplitudes of the computational basis's states that $\left|\phi_{2}\right\rangle$ is superimposed in.

respectively, since the probability of observing $|\Psi\rangle$ in a state that belongs to $\left|\Psi_{1}\right\rangle$ is equal to the desired sum $a$. Therefore, after the initialization stage, the QWSA employs the Quantum Amplitude Estimation (QAE) algorithm [12] for estimating the amplitude of the quantum states that belong to $\left|\Psi_{1}\right\rangle$.

3) Controlled $Q$ Operators: The QCR controls the $Q=$ $A P_{0} A^{\dagger} \cdot C N O T$ operators that are applied to the QFR's state $|\Psi\rangle$, where $A^{\dagger}$ is the conjugate transpose of the matrix representation of $A$ [6], $P_{0}$ flips the sign of the amplitudes of all quantum states except for the all-zero state [6] and $C N O T$ is a Controlled NOT operator that flips the sign of the amplitudes of the states in $|\Psi\rangle$, when the last qubit of those states is equal to $|1\rangle$, therefore marking the wanted states. Every single application of the $Q$ operator to $|\Psi\rangle$ rotates it by $2 \theta$, where we have:

$$
\theta=\arcsin \sqrt{a} \Rightarrow a=\sin ^{2} \theta .
$$

It has been shown [2], [6], [7] that $|\Psi\rangle$ of (10) is a scaled version of the eigenvectors of the $Q$ operator. Therefore the eigenvalues $\lambda_{ \pm}$of $Q$ are connected to the rotation angle $\theta$ through $\lambda_{ \pm}=e^{ \pm j 2 \theta}$. The eigenvalues of $Q$ that are not equal to \pm 1 in our scenario are $\lambda_{ \pm}=-0.55 \pm j 0.84$. Hence we may derive that $\theta=-\frac{j}{2} \ln \left(\lambda_{+}\right)=1.08$. It should be noted that $\lambda_{ \pm}$and $\theta$ is unknown to us in a practical application and we may not exploit it in the simulations, but only for comparison with the estimated value. Furthermore, since $|\Psi\rangle$ consists of the eigenvectors of $Q$, the QFR's states may be considered unaltered and the rotation is performed on the QCR's states instead, as shown in Fig. 2. The quantum system's state after the application of the controlled $Q$ operators becomes

$$
\begin{aligned}
\left|\phi_{1}\right\rangle= & \frac{e^{-j \theta}}{\sqrt{2^{l+2}(1-a)}} \sum_{q=0}^{2^{l}-1} \sum_{x=0}^{N-1}\left(e^{j(2 q+1) \theta}+e^{-j(2 q+1) \theta}\right) . \\
& \cdot \sqrt{P(x)(1-f(x))}|q\rangle|x\rangle|0\rangle+ \\
& +\frac{e^{j \theta}}{\sqrt{2^{l+2} a}} \sum_{q=0}^{2^{l}-1} \sum_{x=0}^{N-1}\left(e^{j(2 q+1) \theta}-e^{-j(2 q+1) \theta}\right) . \\
& \cdot \sqrt{P(x) f(x)}|q\rangle|x\rangle|1\rangle,
\end{aligned}
$$

where $\left|\phi_{1}\right\rangle$ consists of $2^{l} \times(N+1)$ states, as depicted in Figure 3 for $N=2, a=0.775, l=8$ qubits and $\theta=1.08$. 


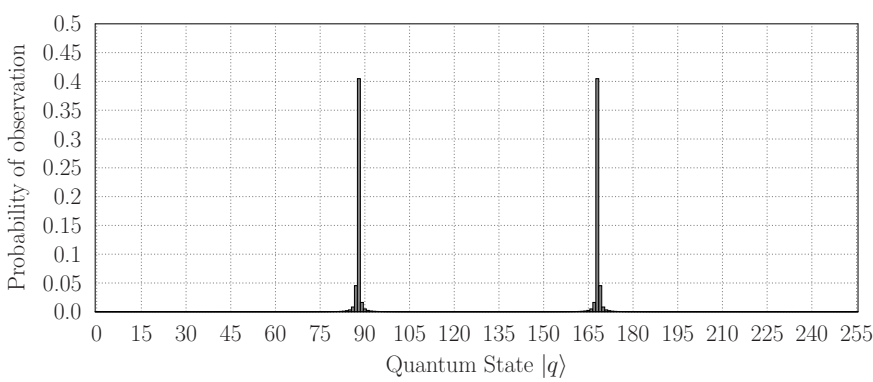

Fig. 5. Probability of observing each of the states in the QCR after the application of the IQFT.

4) Inverse Quantum Fourier Transform (IQFT): For exploiting the periodicity created in the amplitudes of $\left|\phi_{1}\right\rangle$ shown in Fig. 3, the IQFT [2] is applied to $\left|\phi_{1}\right\rangle$. The resultant quantum state $\left|\phi_{2}\right\rangle$ of our system may be described in the computational basis as encapsulated in

$$
\begin{aligned}
\left|\phi_{2}\right\rangle & =\sum_{z=0}^{2^{l}-1} \sum_{x=0}^{N-1} \beta_{z x_{0}}|z\rangle|x\rangle|0\rangle+\sum_{z=0}^{2^{l}-1} \sum_{x=0}^{N-1} \beta_{z x_{1}}|z\rangle|x\rangle|1\rangle \\
\beta_{z x_{1}} & =\frac{\sqrt{P\left(x_{1}\right) f\left(x_{1}\right)}}{2^{l+1} \sqrt{a}} \mu_{z-}, \\
\beta_{z x_{0}} & =\frac{\sqrt{P\left(x_{0}\right)\left(1-f\left(x_{0}\right)\right)}}{2^{l+1} \sqrt{1-a}} \mu_{z+}, \\
\mu_{z \pm} & =e^{j \theta} \frac{1-e^{j 2 \pi\left(2^{l} \frac{\theta}{\pi}-z\right)}}{1-e^{j 2 \pi\left(\frac{\theta}{\pi}-\frac{z}{2^{l}}\right)}} \pm e^{-j \theta} \frac{1-e^{j 2 \pi\left(-2^{l} \frac{\theta}{\pi}-z\right)}}{1-e^{j 2 \pi\left(-\frac{\theta}{\pi}-\frac{z}{2^{l}}\right)}},
\end{aligned}
$$

and the amplitudes of the specific states, which $\left|\phi_{2}\right\rangle$ is superimposed in are illustrated in Fig. 4.

5) Observation of the QCR: Observing only the QCR translates in observing only the first $l=8$ qubits of $\left|\phi_{2}\right\rangle$. The probability of observing the state $\left|z_{o b s}\right\rangle$ in the QCR of Fig. 2 during a partial measurement is the sum of the probabilities of observing the whole system in a state that has the first $l$ qubits equal to $\left|z_{o b s}\right\rangle$. Hence, it is equal to

$$
P\left(z_{o b s}\right)=\sum_{x=0}^{N-1}\left|\beta_{z_{o b s} x_{1}}\right|^{2}+\sum_{x=0}^{N-1}\left|\beta_{z_{o b s} x_{0}}\right|^{2} .
$$

The probability of observing each of the $2^{l}=256$ states in our scenario, where we have $N=4$ is presented in Fig. 5. Due to the IQFT, the probability of observing a state $\left|z_{o b s}\right\rangle$ is equal to the probability of observing the state $\left|2^{l}-z_{o b s}\right\rangle$. In our scenario, by observing the QCR we obtained $\left|z_{o b s}\right\rangle=|88\rangle$.

6) Estimation of $\theta$ and $a$ : The observed state $\left|z_{o b s}\right\rangle=|88\rangle$ provides an estimate of $\theta$ equal to [6], [12]

$$
\hat{\theta}_{z_{o b s}}=\pi \frac{z_{o b s}}{2^{l}}=\pi \frac{88}{256}=1.08 .
$$

Finally, according to (11) the estimated value of the numerator of the LLR in (4) is $\hat{a}=\sin ^{2} \hat{\theta}=0.778$. We would have obtained exactly the same estimate if $\left|z_{\text {obs }}\right\rangle=|256-88\rangle=$ $|168\rangle$. The estimation error is equal to $|a-\hat{a}|=0.0026$. The error in our scenario is the smallest possible value for $l=8$, since we obtained one of the two most likely quantum

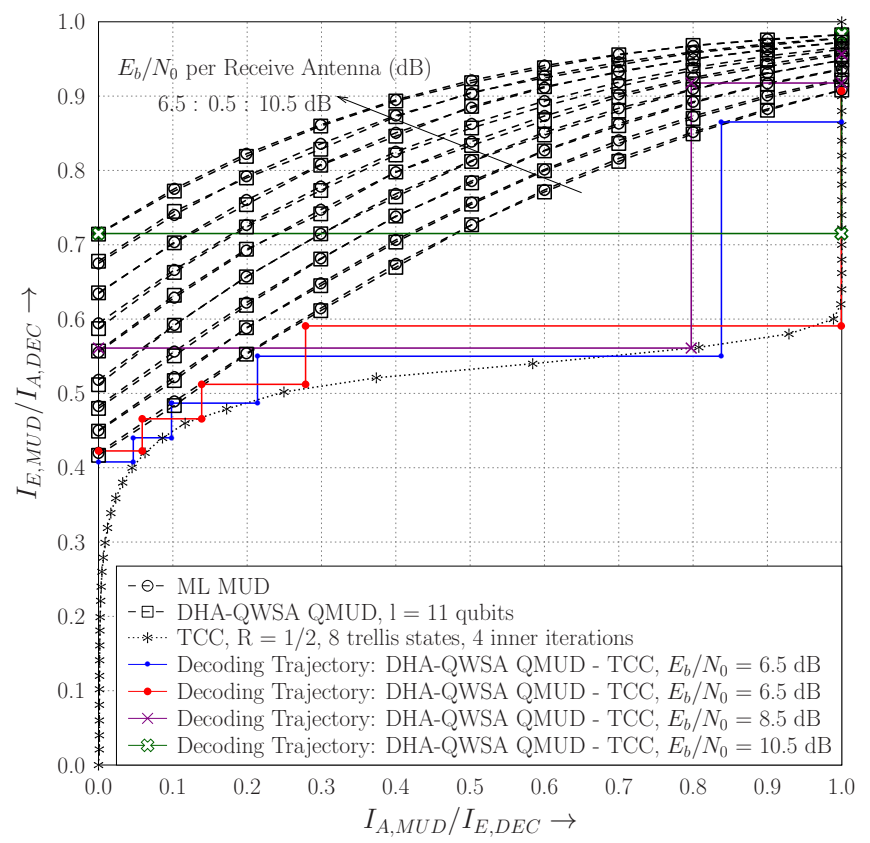

Fig. 6. EXIT chart of an SDMA system supporting $K=3$ users transmitting QPSK symbols with $L=2$ transmit antennas each and $P=3$ receive antennas, TCC with $R=1 / 2,8$ trellis states and $I_{\text {inner }}=4$ inner iterations. DHA-QWSA QMUD is used with $l=11$ qubits in the QCR of Fig. 2.

states, when we observed the QCR, which corresponds to the best estimate of $a$. If more qubits were used in the QCR, the estimation error would be smaller, as encapsulated in [7]

$$
|\hat{a}-a| \leq 2 \pi w \frac{\sqrt{a(1-a)}}{2^{l}}+w^{2} \frac{\pi^{2}}{2^{2 l}}, w=1,2, \ldots
$$

with a probability $8 / \pi^{2}$ for $w=1$ and at least $1-1 /(2(w-1))$ for $w \geq 2$.

\section{Complexity and Simulation Results}

The QWSA has a fixed computational complexity of $2^{l+2}$ CFEs in the QD [6], while the DHA has a variable complexity associated with lower and upper limits of $4.5 \sqrt{M^{K \cdot L}}$ CFEs and $22.5 \sqrt{M^{K \cdot L}}$ CFEs, respectively. In our proposed DHAQWSA QMUD, the DHA is performed only once before the first MUD-decoder iteration for each $\left(M^{K \cdot L}\right)$-ary symbol. The QWSA is performed twice for each bit of the multi-level symbol during every MUD-decoder iteration, therefore it is performed $J \cdot 2 \cdot \log _{2}\left(M^{K \cdot L}\right)$ times for each $\left(M^{K \cdot L}\right)$-ary symbol. In total, the complexity of the DHA-QWSA QMUD per multi-level symbol is [6]

$$
2^{l+3} \cdot J \cdot \log _{2}\left(M^{K \cdot L}\right)+ \begin{cases}22.5 \sqrt{M^{K \cdot L}} & \text { upper bound } \\ 4.5 \sqrt{M^{K \cdot L}} & \text { lower bound }\end{cases}
$$

CFEs, while the complexity of the ML MUD is $M^{K \cdot L}$ CFEs.

Let us now proceed by applying the DHA-QWSA QMUD in an SDMA uplink scenario supporting $K=3$ users, each having $L=2$ TAs and transmitting QPSK $(M=4)$ symbols, while the BS has as few as $P=3$ RAs. Hence the system is rank-deficient, since we have $(K \cdot L) / P=2>1$ and therefore 


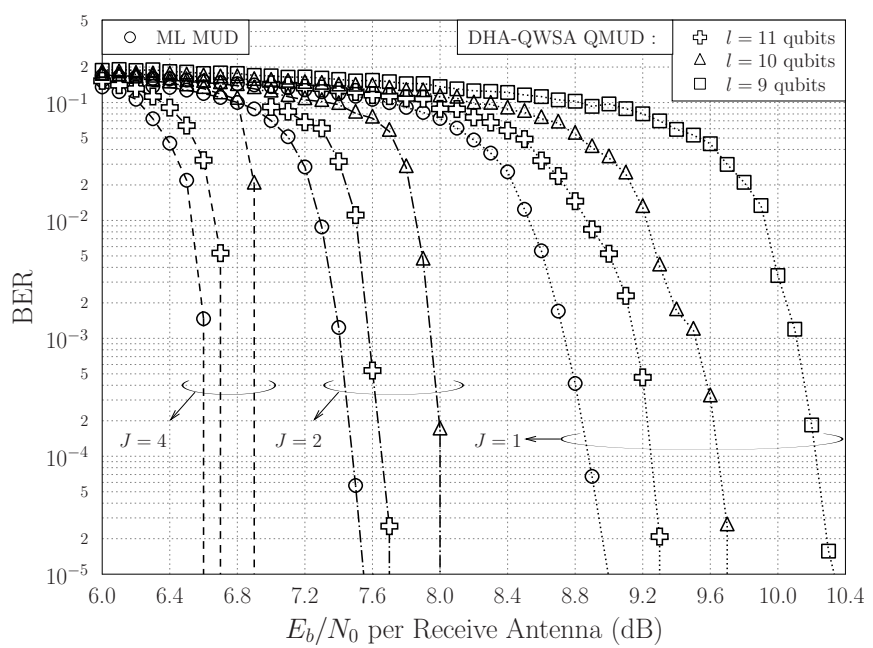

Fig. 7. BER performance of an SDMA system supporting $K=3$ users using QPSK with $L=2$ transmit antennas each and $P=3$ receive antennas, TCC with $R=1 / 2,8$ trellis states and $I=4$ inner iterations for $J=1,2,4$ MUD-Decoder iterations and $l=9,10,11$ qubits in the QCR of Fig. 2. The bit-based interleavers have a length of 10000 bits each.

conventional MUDs such as the MMSE MUD exhibit a high error-floor. TCC with $R=1 / 2$, having 8 trellis states and $I_{\text {inner }}=4$ inner iterations is employed. The EXIT chart of our system employing $l=11$ qubits in the QCR of the QWSA is depicted in Fig. 6 for various values of $E_{b} / N_{0}$ per RA. It should be noted that the EXIT curves of the inner DHAQWSA QMUD are identical to those of the ML MUD for all the $E_{b} / N_{0}$ per RA values. Furthermore, the stair-case-shaped Monte-Carlo simulation-based decoding trajectories verify that the DHA-QWSA QMUD may indeed be readily integrated with iterative receivers.

The BER performance of our SDMA uplink is shown in Fig. 7. The number $J$ of MUD-decoder iterations was varied for $J=1,2,4$, and so was the number of qubits in the QCR for $l=9,10,11$ for the sake of investigating their effect on the BER. Upon increasing the number of qubits $l$ in the QCR, the BER performance of the DHA-QWSA QMUD approaches that of the classic ML MUD, since the accuracy of the QWSA in (16) becomes better. At the same time, the complexity of the QWSA is increased. Moreover, if we increase $J$ while considering $l$ to be fixed, the $E_{b} / N_{0}$ power-loss of the DHAQWSA QMUD with respect to the optimal ML MUD becomes smaller, since the powerful TCC corrects more errors at the output of the QMUD, when $J$ is increased. For $l=11$ qubits, the BER performance of the DHA-QWSA QMUD is just 0.15 $\mathrm{dB}$ away from the optimal limit. If a higher value of $l$ was used, the performance would be expected to be even closer to that of the ML MUD.

The complexity of the ML MUD in our system is $M^{K \cdot L}=$ 4096 CFEs per multi-level symbol. The complexity of the QWSA for $l=11$ is equal to $8192 \mathrm{CFEs}$, hence the application of the QWSA $J \cdot 2 \cdot \log _{2}\left(M^{K \cdot L}\right)$ times for $J=4$ results in a complexity of 786432 CFEs. The average complexity of the DHA in our system was found to be $533.52=8.3 \sqrt{M^{K \cdot L}}$ CFEs. Therefore, the DHA-QWSA QMUD associated with $l=11$ and $J=4$ needed 786966 CFEs for each multilevel symbol. Even though the complexity of the QMUD is higher than that of the ML MUD in this small-scale system, the proposed DHA-QWSA QMUD is designed for large-scale multi-user systems, as shown in [6]. For example, in an SDMA system supporting $K=6$ users transmitting QPSK symbols associated with $M=4, L=2$ TAs per user as well as $J=2$ MUD-decoder iterations, the complexity of the ML MUD is 16777216 CFEs. At the same time, the DHA-QWSA QMUD needs 878592 CFEs, when $l=11$ qubits are used in the QCR for the worst case scenario, where the DHA finds $x_{\max }$ after $22.5 \sqrt{M^{K \cdot L}}=92160$ CFEs.

\section{CONCLUSiOns}

We investigated the application of the DHA-QWSA QMUD in an SDMA uplink scenario, focusing our attention on the functions of the QWSA, while providing the design rationale behind each step of the algorithm as well as behind the architecture of our quantum system. Moreover, we applied the DHA-QWSA QMUD in a rank-deficient SDMA system, where conventional detectors would exhibit a high error-floor and demonstrated that it offers a near-ML performance both with the aid of EXIT charts and BER curves.

\section{REFERENCES}

[1] L. Hanzo, O. Alamri, M. El-Hajjar and N. Wu, Near-Capacity MultiFunctional MIMO Systems: Sphere-Packing, Iterative Detection and Cooperation. Chichester, UK: John Wiley \& Sons, IEEE Press, May 2009.

[2] M. A. Nielsen and I. L. Chuang, Quantum Computation and Quantum Information. Cambridge University Press, 2000.

[3] S. Imre and F. Balázs, Quantum Computing and Communications: An Engineering Approach. John Wiley \& Sons, 2005.

[4] S. Imre and L. Gyongyosi, Advanced Quantum Communications: An Engineering Approach. John Wiley \& Sons, 2013.

[5] S. Imre and F. Balázs, "Non-Coherent Multi-user Detection Based on Quantum Search," in ICC 2002. IEEE International Conference on Communications, vol. 1, pp. 283-287, 2002.

[6] P. Botsinis, S. X. Ng, and L. Hanzo, "Quantum Search Algorithms, Quantum Wireless, and a Low-Complexity Maximum Likelihood Iterative Quantum Multi-User Detector Design," IEEE Access, vol. 1, pp. 94-122, 2013. http://ieeexplore.ieee.org/xpl/articleDetails.jsp?arnumber=6515077.

[7] G. Brassard, F. Dupuis, S. Gambs, and A. Tapp, "An Optimal Quantum Algorithm to Approximate the Mean and its Application for Approximating the Median of a Set of Points Over an Arbitrary Distance," eprint arXiv:quant-ph/1106.4267v1, June 2011.

[8] C. Durr and P. Høyer, "A Quantum Algorithm for Finding the Minimum," eprint arXiv:quant-ph/9607014, July 1996.

[9] L. K. Grover, "A Fast Quantum Mechanical Algorithm for Database Search," Proceedings, 28th Annual ACM Symposium on the Theory of Computing, pp. 212-219, May 1996.

[10] M. Boyer, G. Brassard, P. Høyer, and A. Tapp, "Tight Bounds on Quantum Searching," Fortschritte der Physik, vol. 46, pp. 493-506, 1998.

[11] L. Hanzo, T. H. Liew, B. Yeap, R. Y. S. Tee, and S. X. Ng, Turbo Coding, Turbo Equalisation and Space-Time Coding: EXIT-Chart Aided Near-Capacity Designs for Wireless Channels. John Wiley \& Sons, 2010.

[12] G. Brassard, P. Høyer, M. Mosca, and A. Tapp, "Quantum Amplitude Amplification and Estimation," eprint arXiv:quant-ph/0005055, May 2000. 